\title{
COMMON FIXED POINTS OF MAPS ON FUZZY METRIC SPACES
}

\author{
S.N. MISHRA \\ Department of Mathematics \\ University of Transkei \\ Private Bag X1, UMTATA \\ Republic of Transkei, Southern Africa \\ and \\ NILIMA SHARMA \\ S.L. SINGH \\ Department of Mathematics \\ Gurukula Kangri University \\ Hardwar 249404, India
}

(Received December 30, 1992 and in revised form February 15, 1993)

\begin{abstract}
Following Grabiec's approach to fuzzy contraction principle, the purpose of this note is to obtain common fixed point theorems for asymptotically commuting maps on fuzzy metric spaces.
\end{abstract}

KEY WORDS AND PHRASES. Fuzzy metric space (FM-space), fixed point, contraction, contractive, asymptotically commuting maps, compatible maps.

1991 AMS SUBJECT CLASSIFICATION CODES. 54H25, 54A40, 54E99.

\section{INTRODUCTION.}

L. Zadeh's investigation [19] of the notion of fuzzy sets has led to a rich growth of fuzzy mathematics (see [13]). Now it is a well recognized system to embrace upon uncertanties arising in various physical situations. The theory of fixed point equations is one of the preeminent basic tools to handle various physical formulations. Fixed point theorems in fuzzy mathematics are emerging with vigorous hope and vital trust. There have been several attempts to formulate fixed point theorems in fuzzy mathematics (see, for instance, [1]-[3] and [18]). However, it appears that Kramosil and Michálek's study [8] of fuzzy metric spaces (FM-spaces) paves a way for very soothing machinery to develop fixed point theorems especially for contractive type maps. From amongst several (at least four) formulations of FM-spaces, Grabiec [3] followed KramosilMichálek [8] and obtained the fuzzy version of Banach contraction principle. This paper offers fixed point theorems on FM-spaces, which generalize, extend and fuzzify several known fixed point theorems for contractive type maps on metric and other spaces. One of its corollaries and Grabiec's fuzzy Banach contraction theorem are applied to obtain a fixed point type theorem on the product of an FM-space.

\section{PRELIMINARIES.}

Following Grabiec [3] and Kramosil-Machálek [8], we follow the following notations and definitions. 
A fuzzy metric space (FM-space) is an ordered triplet $(X, M, *)$ consisting of a nonempty set $X$, a fuzzy set $M$ in $X^{2} \times[0, \infty)$ and *, a continuous $T$-norm [11] (see also [3], [8]). The functions $M(x, y, \cdot):[0, \infty) \rightarrow[0,1]$ are left-continuous and are assumed to satisfy the following conditions:

(FM-1) $M(x, y, t)=1$ for all $t>0$ iff $x=y$,

(FM-2) $M(x, y, 0)=0$,

(FM-3) $M(x, y, t)=M(y, x, t)$,

(FM-4) $M(x, y, t) * M(y, z, s) \leq M(x, z, t+s)$

for all $x, y, z$ in $X$ and $t, s \geq 0$.

Grabiec [3] has shown that $M(x, y, \cdot)$ is nondecreasing for all $x, y$ in $X$. Refer [3] and [8] for topological preliminaries.

In all that follows $N$ stands for the set of natural numbers and $X$ stands for an FM-space $(X, M, *)$ with the following property:

(FM-5) $\lim _{t \rightarrow \infty} M(x, y, t)=1$ for all $x, y$ in $X$.

Proof of the following lemma is implicated in ([3], Th. 5).

LEMMA 1. Let $\left\{y_{n}\right\}$ be a sequence in an FM-space $X$. If there exists a positive number $k<1$ such that

$$
M\left(y_{n+2}, y_{n+1}, k t\right) \geq M\left(y_{n+1}, y_{n}, t\right), t>0, n \in N,
$$

then $\left\{y_{n}\right\}$ is a Cauchy sequence.

We shall use the following fact generally without reference to it.

LEMMA 2. If for two points $x, y$ of $X$ and for a positive number $k<1$,

$$
M(x, y, k t) \geq M(x, y, t)
$$

then $x=y$.

PROOF. It is immediate from (FM-5).

\section{ASYMPTOTICALLY COMMUTING MAPS AND COMMON FIXED POINTS.}

DEFINITION 1. Self-maps $P$ and $S$ of an FM-space $X$ will be called $z$-asymptotically commuting (or simply asymptotically commuting) iff for all $t>0$

$$
\lim _{n \rightarrow \infty} M\left(P S x_{n}, S P x_{n}, t\right)=1
$$

whenever $\left\{x_{n}\right\}$ is a sequence in $X$ such that

$$
\lim _{n \rightarrow \infty} P x_{n}=\lim _{n \rightarrow \infty} S x_{n}=z \text { for some } z \text { in } X .
$$

For an equivalent formulation in a metric space refer to Jungck ([6]-[7]) and Trivari-Singh [17]. Following Jungck's nomenclature ([6]-[7]), asymptotically commuting maps may also be called compatible maps. Such maps are more general than commuting and weakly commuting maps [12] (see also [6]) both.

LEMMA 3. If $Q$ and $T$ are asymptotically commuting maps on an FM-space $X$ with $t * t \geq t$ for all $t \in[0,1]$ and $Q w_{n}, T w_{n} \rightarrow z$ for some $z$ in $X,\left(w_{n}\right.$ being a sequence in $\left.X\right)$ then $Q T w_{n} \rightarrow T z$ provided $T$ is continuous (at $z$ ). 
PROOF. By the continuity of $T$ at $z, T Q w_{n} \rightarrow T z$ and $T T w_{n} \rightarrow T z$. By the asymptotic commutativity of $\{Q, T\}$.

$$
M\left(Q T w_{n}, T Q w_{n}, t\right) \rightarrow 1 \text { as } n \rightarrow \infty
$$

Therefore

$$
M\left(T z, Q T w_{n}, t\right) \geq M\left(T z, T Q w_{n}, t / 2\right) * M\left(T Q w_{n}, Q T w_{n}, t / 2\right)
$$

yields

$$
\lim _{n \rightarrow \infty} M\left(T z, Q T w_{n}, t\right) \geq 1 * 1=1
$$

This proves the lemma.

For an equivalent formulation of Lemmas 3 on a metric space, refer Jungck [6].

THEOREM 1. Let $(X, M, *)$ be a complete FM-space with $t * t \geq t, t \in[0,1]$, and $P, Q: X \rightarrow X$. If there exist continuous maps $S, T: X \rightarrow X$ and a constant $k \in(0,1)$ such that

$$
S T=T S,
$$

$\{P, S\}$ and $\{Q, T\}$ are asymptotically commuting pairs,

$$
P T(X) \cup Q S(X) \subset S T(X) \text {, and }
$$

$$
\begin{gathered}
M(P x, Q y, k t) \geq M(S x, T y, t) * M(P x, S x, t) * M(Q y, T Y, t) \\
* M(P x, T y, \alpha t) * M(Q y, S x,(2-\alpha) t)
\end{gathered}
$$

for all $x, y$ in $X, t>0$ and $\alpha \in(0,2)$; then $P, Q, S$ and $T$ have a unique common fixed point.

PROOF. Pick $x_{0} \in X$. Construct a sequence $\left\{x_{n}\right\}$ as follows:

$$
P T x_{2 n}=S T x_{2 n+1}, Q S X_{2 n+1}=S T x_{2 n+2}, n=0,1,2, \cdots .
$$

We can do this since (3.3) holds. Indeed such a sequence was first introduced in ([15]-[16]).

Let $z_{n}=S T x_{n}$. Then, for $\alpha=1-q, q \in(0,1)$, by (3.4),

$$
\begin{gathered}
M\left(z_{2 n+1}, z_{2 n+2}, k t\right)=M\left(P T x_{2 n}, Q S x_{2 n+1}, k t\right) \\
\geq M\left(z_{2 n}, z_{2 n+1}, t\right) * M\left(z_{2 n+1}, z_{2 n}, t\right) * M\left(z_{2 n+2}, z_{2 n+1}, t\right) * M\left(z_{2 n+1},\right. \\
\left.z_{2 n+1},(1-q) t\right) * M\left(z_{2 n+2}, z_{2 n},(1+q) t\right) \\
\geq M\left(z_{2 n}, z_{2 n+1}, t\right) * M\left(z_{2 n+1}, z_{2 n+2}, t\right) * M\left(z_{2 n}, z_{2 n+1}, q t\right) .
\end{gathered}
$$

Since the norm * is continuous and $M(x, y, \cdot)$ is left-continuous, making $q \rightarrow 1$ gives

$$
M\left(z_{2 n+1}, z_{2 n+2}, k t\right) \geq M\left(z_{2 n}, z_{2 n+1}, t\right) * M\left(z_{2 n+1}, z_{2 n+2}, t\right) .
$$

Similarly, taking $x=T x_{2 n+2}, y=S x_{2 n+1}, \alpha=1+q^{\prime}, q^{\prime} \in(0,1)$ in (3.4), simplifying and making $q^{\prime} \rightarrow 1$, we have

So in general

$$
M\left(3_{2 n+2}, 3_{2 n+3}, k t\right) \geq M\left(3_{2 n+1}, 3_{2 n+2}, t\right) * M\left(3_{2 n+2}, 3_{2 n+3}, t\right) .
$$

Consequently,

$$
M\left(z_{m+1}, z_{m+2}, k t\right) \geq M\left(z_{m}, z_{m+1}, t\right) * M\left(z_{m+1}, z_{m+2}, t\right), m \in N .
$$

$$
M\left(z_{m+1}, z_{m+2}, k t\right) \geq M\left(z_{m}, z_{m+1}, t\right) * M\left(z_{m+1}, z_{m+2}, t k^{-p}\right), m, p \in N
$$


Since $M\left(z_{m+1}, z_{m+2}, t k^{-p}\right) \rightarrow 1$ as $p \rightarrow \infty$, we have

$$
M\left(z_{m+1}, z_{m+2}, k t\right) \geq M\left(z_{m}, z_{m+1}, t\right), m \in N .
$$

By Lemma 1, $\left\{z_{n}\right\}$ is a Cauchy sequence, and has a limit in $X$. Call it $z$. $\left\{P T x_{2 n}\right\}$ and $\left\{Q S x_{2 n+1}\right\}$ being the subsequences of $\left\{S T x_{n}\right\}$ also converge to $z$.

Let $y_{n}=T x_{n}$ and $w_{n}=S x_{n}, n \in N$. Then $P y_{2 n} \rightarrow z, S y_{2 n} \rightarrow z, T w_{2 n+1} \rightarrow z$ and $Q w_{2 n+1} \rightarrow z$. So, for $t>0$, by (3.2),

$$
M\left(P S y_{2 n}, S P y_{2 n}, t\right) \rightarrow 1 \text { and } M\left(Q T w_{2 n+1}, T Q w_{2 n+1}, t\right) \rightarrow 1 .
$$

Moreover, by the continuity of $T$ and Lemma 3,

$$
T T w_{2 n+1} \rightarrow T z, T Q w_{2 n+1} \rightarrow T z \text { and } Q T w_{2 n+1} \rightarrow T z .
$$

By (3.4) with $\alpha=1$,

This yields

$$
\begin{gathered}
M\left(P y_{2 n}, Q T w_{2 n+1}, k t\right) \geq M\left(S y_{2 n}, T T w_{2 n+1}, t\right) * M\left(P y_{2 n}\right. \\
\left.S y_{2 n}, t\right) * M\left(Q T w_{2 n+1}, T T w_{2 n+1}, t\right) * M\left(P y_{2 n}, T T w_{2 n+1}\right. \\
t) * M\left(Q T w_{2 n+1}, S y_{2 n}, t\right)
\end{gathered}
$$

$$
M(z, T z, k t) \geq M(z, T z, t) * 1 * 1 * M(z, T z, t) * M(T z, z, t) .
$$

So $z=T z$. Similarly $z=S z$.

Again by (3.4) with $\alpha=1$,

$$
\begin{gathered}
M\left(P y_{2 n}, Q z, k t\right) \geq M\left(S y_{2 n}, T z, t\right) * M\left(P y_{2 n}, S y_{2 n}, t\right) * M(Q z \\
T z, t) * M\left(P y_{2 n}, T z, t\right) * M\left(Q z, S y_{2 n}, t\right)
\end{gathered}
$$

yields

$$
M(z, Q z, k t) \geq 1 * 1 * M(Q z, z, t) * 1 * M(Q z, z, t) .
$$

So $Q z=z$. Similarly $P z=z$.

The uniqueness of $z$ as the common fixed point of $P, Q, S$ and $T$ follows easily from (3.4).

REMARK 1. If $S=T$ and $P=Q$, then (3.1)-(3.3) say that $P$ and $S$ are asymptotically commuting and $P(X) \subset S(X)$. In such a situation, the sequence $\left\{S T x_{n}\right\}$ constructed in the proof of Theorem 1 reduces to $\left\{P x_{n}=S x_{n+1}\right\}$, and such a sequence was first introduced by Jungck [5].

REMARK 2. If $S=T$ then (3.1)-(3.3) say that $S$ is asymptotically commuting with each of $P, Q$, and $P(X) \cup Q(X) \subset S(X)$. In this situation, the sequence $\left\{S T x_{n}\right\}$ is replaced by $\left\{S x_{n}\right\}$ wherein $P x_{2 n}=S x_{2 n+1}$ and $Q x_{2 n+1}=S x_{2 n+2}$, which was first introduced by Singh [14].

Theorem 1 with $S=T=$ the identity map is:

COROLLARY 1. Let $(X, M, *)$ be a complete FM-space with $t * t \geq t, t \in[0,1]$, and $P, Q: X \rightarrow X$. If there exists a constant $k \in(0,1)$ such that

(C-1) $M(P x, Q y, k t) \geq$

$$
M(x, y, t) * M(x, P x, t) * M(y, Q y, t) * M(y, P x, t) * M(x, Q y,(2-\alpha) t)
$$

for all $x, y$ in $X, t>0$ and $\alpha \in(0,2)$, then $P$ and $Q$ have a unique common fixed point. 
In view of Remark 1 we have the following result.

COROLLARY 2. Let $(X, M, *)$ be a complete FM-space with $t * t \geq t, t \in[0,1]$, and $P, S$ asymptotically commuting maps on $X$ such that $P(X) \subset S(X)$. If $S$ is continuous and there exists a constant $k \in(0,1)$ such that

(C-2) $M(P x, P y, t) \geq$ $M(S x, S y, t) * M(S x, P x, t) * M(S y, P y, t) * M(S y, P x, \alpha t) * M(S x, P y,(2-\alpha) t)$

for all $x, y$ in $X, t>0$ and $\alpha \in(0,2)$, then $P$ and $S$ have a unique common fixed point.

COROLLARY 3. (Grabiec's fuzzy Banach contraction theorem (see [3])). Let $X$ be a complete FM-space with $t * t \geq t, t \in[0,1]$, and $P: X \rightarrow X$ such that

(C-3) $M(P x, P y, k t) \geq M(x, y, t)$

for all $x, y$ in $X ; 0<k<1$. Then $P$ has a unique fixed point.

PROOF. It follows from Corollary 1 since (C-1) with $P=Q$ includes (C-3). However, Grabiec [3] does not require " $t * t \geq t$ " in his proof.

The above fixed point theorems extend, generalize and fuzzify several fixed point theorems on metric, Menger and uniform spaces (cf. [3]-[7], [9]-[10], [14]-[17]). The following metric version of the condition (C-1) is perhaps enough to elaborate this remark. For self-maps $P$ and $Q$ of a metric space $(Y, d)$ and $0<k<1$, the metric version of $(\mathrm{C}-1)$ is:

$$
d(P x, Q y) \leq k \max \{d(x, y), d(x, P x), d(y, Q y),[d(y, P x)+d(x, Q y)] / 2\}
$$

for all $x, y$ in $Y$.

\section{AN APPLICATION.}

Now we shall apply Corollaries 1 and 3 to establish the following result.

THEOREM 2. Let $(X, M, *)$ be a complete FM-space with $t * t \geq 0, t \in[0,1]$, and $P, Q$ two maps on the product $X \times X$ with values in $X$. If there exists a constant $k \in(0,1)$ such that

$$
\begin{gathered}
M(P(x, y), Q(u, v), k t) \geq \\
M(P(x, y), x, t) * M(Q(u, v), u, t) * M(x, u, t) * M(y, v, t) \\
* M(P(x, y), u, \alpha t) * M(Q(u, v), x,(2-\alpha) t)
\end{gathered}
$$

for all $x, y, u, v$ in $X, t>0$ and $\alpha \in(0,2)$, then there exists exactly one point $w$ in $X$ such that

$$
P(w, w)=w=Q(w, w) .
$$

PROOF. By (4.1),

$$
\begin{gathered}
M(P(x, y), Q(u, y), t) \geq \\
M(P(x, y), x, t) * M(Q(u, y), u, t) * M(x, u, t) * M(P(x, y), u, \alpha t) * M(Q(u, y), x,(2-\alpha) t)
\end{gathered}
$$

for all $x, y, u$ in $X$. Therefore, by Corollary 1 , for each $y$ in $X$, there exists one and only one $z(y)$ in $X$ such that

For any $y, y^{\prime} \in X$, by (4.1) with $\alpha=1(z(y), y)=z(y)=Q(z(y), y)$.

that is

$$
\begin{gathered}
M\left(z(y), z\left(y^{\prime}\right), k t\right)=M\left(P(z(y), y), Q\left(z\left(y^{\prime}\right), y\right), t\right) \\
\geq 1 * 1 * 1 * M\left(y, y^{\prime}, t\right) * M\left(z(y), z\left(y^{\prime}\right), t\right) * M\left(z(y), z\left(y^{\prime}\right), t\right),
\end{gathered}
$$

$$
M\left(z(y), z\left(y^{\prime}\right), k t\right) \geq M\left(y, y^{\prime}, t\right) * M\left(z(y), z\left(y^{\prime}\right), t\right)
$$




$$
\begin{aligned}
& \geq M\left(y, y^{\prime}, t\right) * M\left(z(y), z,\left(y^{\prime}\right), t / k^{n}\right) \\
& \rightarrow M\left(y, y^{\prime}, t\right) * 1 .
\end{aligned}
$$

So

$$
M\left(z(y), z\left(y^{\prime}\right), k t\right) \geq M\left(y, y^{\prime}, t\right) .
$$

Therefore Corollary 3 yields that the map $z(\cdot)$ of $X$ into itself has exactly one fixed point $w$ in $X$, i.e., $z(w)=w$. Hence by $(4.2), w=z(w)=P(w, w)=Q(w, w)$. It is easily seen that $P$ and $Q$ can have only one such point $w$ in $X$.

REMARK 3. If $x, y \in X$ are such that $x=P(x, y)$ and $y=Q(x, y)$ then it can be seen using (4.1) that $x=y$. These results are essentially motivated by the work of Professor Kiyoshi Iséki [4].

\section{REFERENCES}

1. BADARD, R., Fixed point theorems for fuzzy numbers, Fuzzy Sets Systems 13 (1984). 291302.

2. BUTNARIU, D., Fixed points for Fuzzy mappings, Fuzzy Sets Systems 7 (1982), 191-207.

3. GRABIEC, M., Fixed points in fuzzy metric spaces, Fuzzy Sets Systems 27 (1988), 385-389.

4. ISEḰI, K., Some applications of Banach type contraction principles, Math. Sem. Notes Kobe Univ. 4 (1976), 211-214.

5. JUNGCK, G., Commuting mappings and fixed points, Amer. Math. Monthly 83 (1976), 261-263.

6. JUNGCK, G., Compatible mappings and common fixed points, Internat. J. Math. and Math. Sci. 9 (1986), 771-779.

7. JUNGCK, G., Common fixed points for commuting and compatible maps on compacta, Proc. Amer. Math. Soc. 103 (1988), 977-983.

8. KRAMOSIL, I. \& MICHÁLEK, J., Fuzzy metric and statistical metric spaces, Kybernetika 11 (1975), 336-344.

9. RHOADES, B.E., A comparison of various definitions of contractive mappings, Trans. Amer. Math. Soc. 226 (1977), 257-290.

10. RHOADES, B.E., Contractive definitions revisited, Contemporary Mathematics 21 (1983), 189-205.

11. SCHWEIZER, B. \& SKLAR, A., Statistical metric spaces, Pac. J. Math. 10 (1960), 313-334.

12. SESSA, S., On a weak commutativity condition of mappings in fixed point considerations Publ. Inst. Math. (Beograd) 32(46) (1982), 149-153.

13. SHOSTAK, A.P., Two decades of fuzzy topology: basic ideas, notions and results, Russian Math. Surveys, 44:6 (1989), 123-186.

14. SINGH, S.L., Some common fixed point theorems in L-spaces, Math. Sem. Notes Kobe Univ. 7 (1979), 91-97.

15. SINGH, S.L. \& KASAHARA, S., On some recent results on common fixed points, Indian J. Pure Appl. Math. 13 (1982), 757-761; Corrigendum 14 (1983), 1075.

16. SINGH, S.L. \& RAM, B., Common fixed points of commuting mappings in 2-metric spaces, Math. Sem. Notes Kobe Univ. 10 (1982), 197-208.

17. TIVARI, B.M.L. \& SINGH, S.L., A note on recent generalizations of Jungck contraction principle, J. UPGC. Acad. Soc. 3 (1986), 13-18.

18. WEISS, M.D., Fixed points, separation and induced topologies for fuzzy sets, J. Math. Anal. Appl. 50 (1975), 142-150.

19. ZADEH, L.A., Fuzzy sets, Inform. Control 8 (1965), 338-353. 


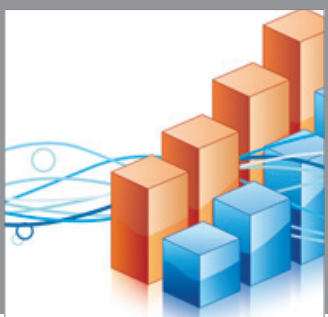

Advances in

Operations Research

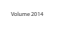

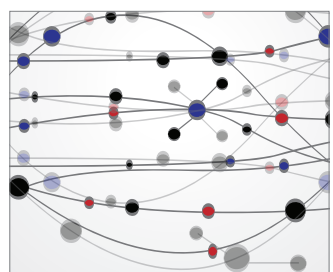

\section{The Scientific} World Journal
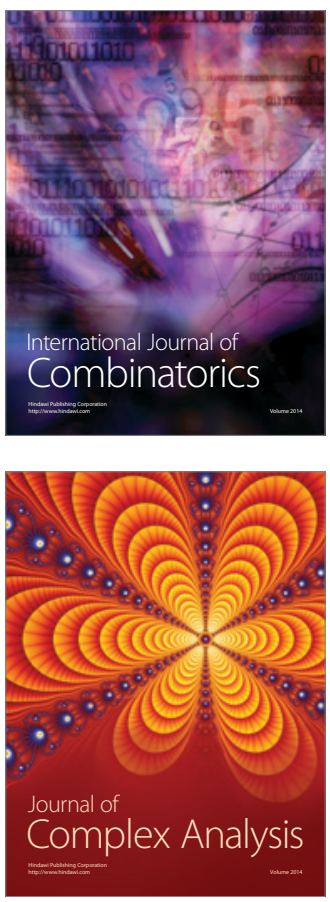

International Journal of

Mathematics and

Mathematical

Sciences
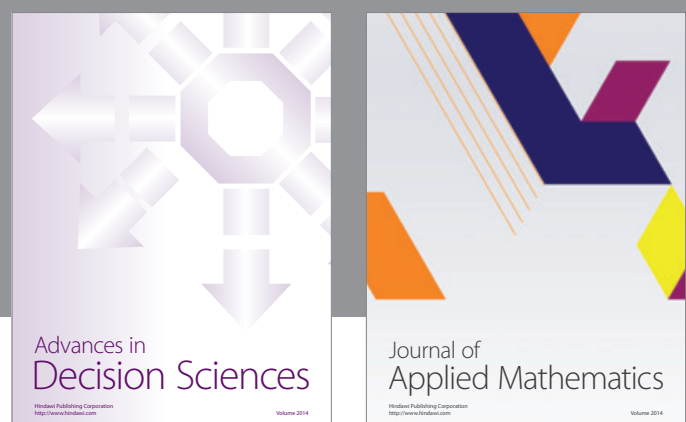

Journal of

Applied Mathematics
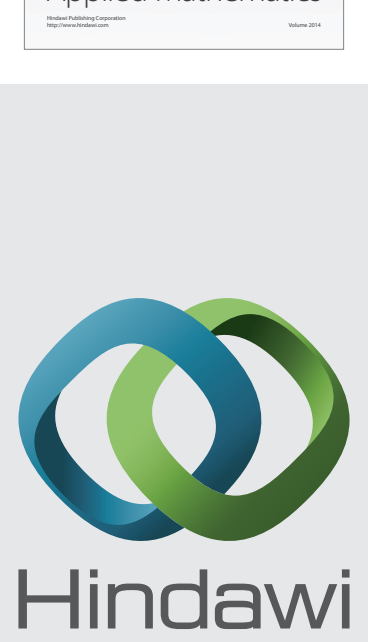

Submit your manuscripts at http://www.hindawi.com
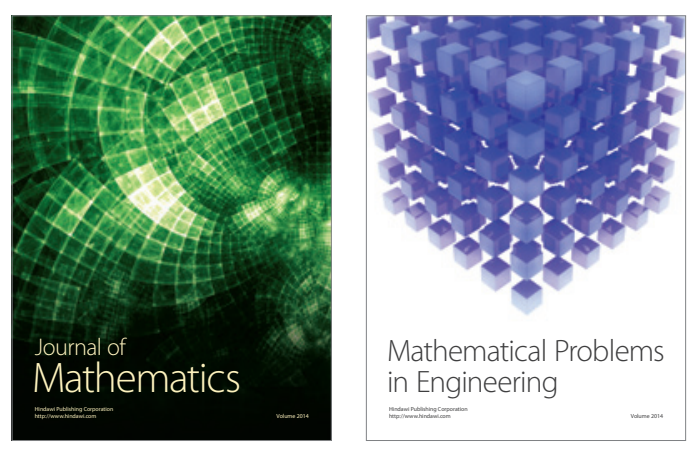

Mathematical Problems in Engineering
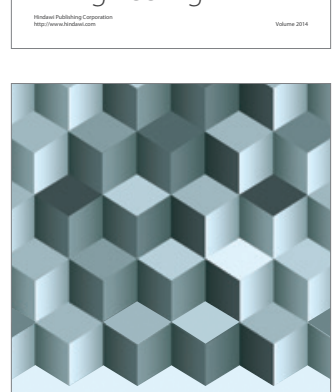

Journal of

Function Spaces
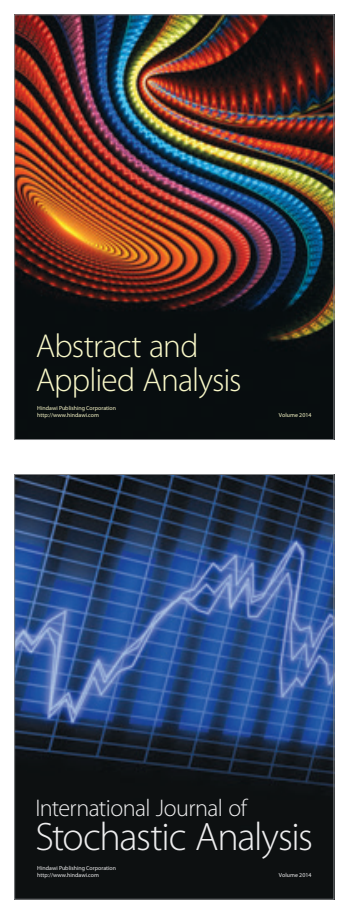

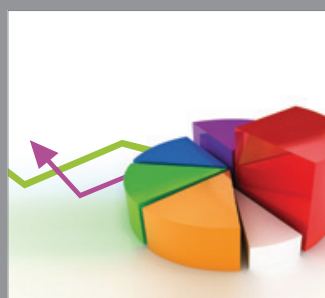

ournal of

Probability and Statistics

Promensencen
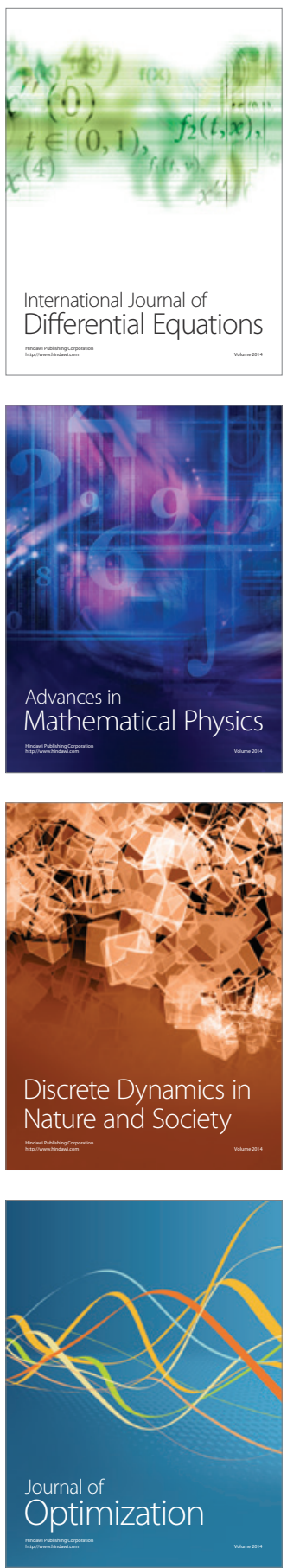